Pacific

Journal of

Mathematics

A FAMILY OF TRIPLY PERIODIC COSTA SURFACES

VALÉRIo RAmos Batista

Volume $212 \quad$ No. 2

December 2003 


\title{
A FAMILY OF TRIPLY PERIODIC COSTA SURFACES
}

\author{
VALÉRIo RAmos BAtista
}

\begin{abstract}
We derive global Weierstrass representations for complete minimal surfaces obtained by substituting the ends of the Costa surface by symmetry curves.
\end{abstract}

\section{Introduction.}

Among all known complete embedded minimal surfaces in $\mathbb{R}^{3}$ the triply periodic ones form the richest class of examples regarding variety of genus and symmetry group. For instance, the gyroid can be pointed out as a complete minimal surface containing neither straight lines nor reflectional symmetry curves. Another curiosity is the associate family from the Schwarz P-Surface till its conjugate, the D-Surface, which has the gyroid as an intermediate embedded member (see [4], p. 25). Another associate family with such a behaviour is unknown outside the triply periodic class.

The variety of triply periodic minimal surfaces has been known much earlier than most of the non-triply periodic examples, even if the existence proof of the formers took longer to be concluded. The first five examples came out in 1890 due to a work of H.A. Schwarz and his students [18]. This work inspired A.H. Schoen [17] who presented 17 other such surfaces in 1970. Later in $1989 \mathrm{H}$. Karcher [9] proved the existence of these triply periodic examples and found many others with the conjugate surface method.

In this paper we enrich this class, not only by presenting a new family of triply periodic minimal surfaces in $\mathbb{R}^{3}$, but also with a full study of this family which includes uniqueness and limits. Such a thorough study is rare to be found among other triply periodic examples. Moreover, in this new family the boundary contour of the conjugate surfaces patch does not project onto any convex domain. Hence, the classical Plateau approach fails for these particular surfaces and they are perhaps the simplest such examples.

In order to find new surfaces one can make use of already known examples like the Costa surface, which gave rise to the " $M_{k}$-Costa-Hoffman-Meeks" families [7], later generalised by D. Hoffman and H. Karcher [5]. In this present paper, once more the Costa surface turns out to be a source of new results. 


\section{Background.}

In this section we state some well-known theorems on minimal surfaces. For details we refer the reader to $[\mathbf{9}],[\mathbf{1 2}]$ and $[\mathbf{1 4}]$. In this paper all surfaces are required to be regular.

Theorem 2.1 (Weierstrass representation). Let $S$ be a minimal surface in $\mathbb{R}^{3}$ and $R$ the underlying Riemann surface of $S$. Let dh be a meromorphic 1 -form on $R$ and $g: R \rightarrow \hat{\mathbb{C}}:=\mathbb{C} \cup \infty$ a meromorphic function. Then $X: R \rightarrow \mathbb{R}^{3}$ given by

$$
X(p):=\frac{1}{2} \operatorname{Re} \int^{p}\left(g-g^{-1}, i\left(g+g^{-1}\right), 2\right) d h
$$

is a conformal regular minimal immersion provided the poles and zeros of $g$ coincide with the zeros of $d h$. Conversely, every regular conformal minimal immersion $X: R \rightarrow \mathbb{R}^{3}$ can be expressed in this form for some meromorphic function $g$ and meromorphic 1-form $d h$.

Definition 2.2. The pair $(g, d h)$ is the Weierstrass data on $R$ of the minimal immersion $X: R \rightarrow X(R)=S \subset \mathbb{R}^{3}$.

Definition 2.3. A complete, orientable minimal surface $S$ is algebraic if it admits a Weierstrass representation such that $R=\bar{R}-\left\{p_{1}, \ldots, p_{r}\right\}$, where $\bar{R}$ is compact and both $g$ and $d h$ extend meromorphically to $\bar{R}$.

Definition 2.4. An end of $S$ is the image of a punctured neighbourhood $V_{p}$ of a point $p \in\left\{p_{1}, \ldots, p_{r}\right\}$ such that $\left(\left\{p_{1}, \ldots, p_{r}\right\}-p\right) \cap \bar{V}_{p}=\emptyset$. The end is embedded if this image is embedded for a sufficiently small neighbourhood of $p$.

Theorem 2.5. Let $S$ be an algebraic minimal surface whose genus of $\bar{R}$ is $k$ and the number of ends is $r$ (all of them embedded). Then

$$
\operatorname{deg}(g)=k+r-1 .
$$

Theorem 2.6. Let $S$ be a complete minimal surface in $\mathbb{R}^{3}$. Then $S$ is algebraic if, and only if, it can be obtained from a piece $S_{t}$ of finite total curvature by applying a finitely generated translation group $G_{t}$ of $\mathbb{R}^{3}$.

From now on we consider only algebraic surfaces. The function $g$ is the stereographic projection of the Gauß map $N: R \rightarrow S^{2}$ of the minimal immersion $X$. This minimal immersion is well-defined in $\mathbb{R}^{3} / G_{t}$, but is allowed to be a multivalued function in $\mathbb{R}^{3}$. The function $g$ is a covering map of $\hat{\mathbb{C}}$, and hence the total curvature of $S_{t}$ is $-4 \pi \operatorname{deg}(g)$.

\section{The Costa surface.}

We describe the Costa surface, which is the starting point of our constructions. Details are found in $[\mathbf{3}]$ and $[\mathbf{6}]$. 
Theorem 3.1 (The Costa surface). Let $\bar{R}$ be the square torus whose algebraic equation is

$$
\wp^{\prime 2}=\wp(1-\wp)(1+\wp) .
$$

For some positive $\mu$, define $g=\mu \wp^{\prime}$ and $d h=\wp d \wp / \wp^{\prime}$. Then there exists a unique positive value of $\mu$ such that $(g, d h)$ is the Weierstrass pair on $R=\bar{R}-\wp^{-1}(\{-1,1, \infty\})$ of a complete minimal embedding of $R$ in $\mathbb{R}^{3}$.

The next picture represents the image of the minimal embedding referred to by the previous theorem.

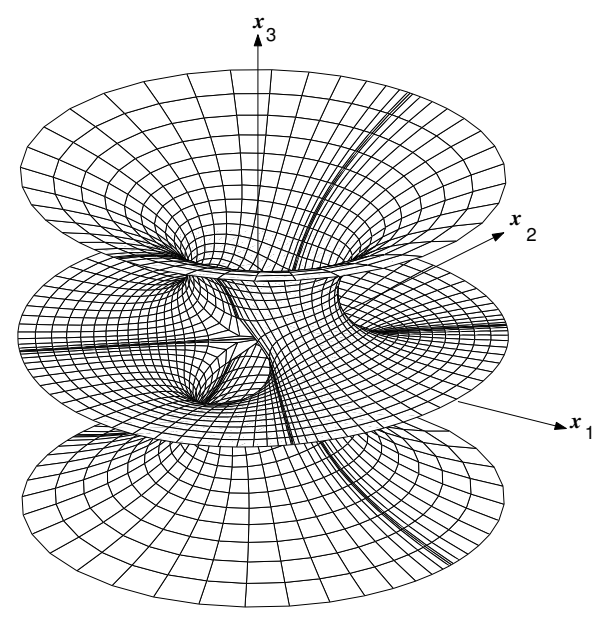

(a)

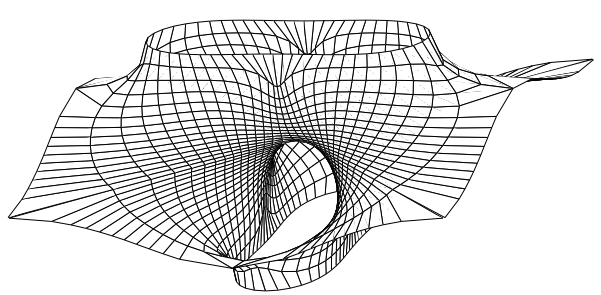

(b)

Figure 1. (a) The Costa surface; (b) the fundamental piece $P$ of a triply periodic Costa surface.

After a suitable rigid motion in $\mathbb{R}^{3}$ we can position the Costa surface in such a way that it will have the following symmetries:

$$
\begin{aligned}
\sigma_{1} & :=\left(x_{1}, x_{2}, x_{3}\right) \rightarrow\left(-x_{1}, x_{2}, x_{3}\right) ; \\
\sigma_{2} & :=\left(x_{1}, x_{2}, x_{3}\right) \rightarrow\left(x_{1},-x_{2}, x_{3}\right) ; \\
\sigma_{3} & :=\left(x_{1}, x_{2}, x_{3}\right) \rightarrow\left(x_{2}, x_{1},-x_{3}\right) \text { and } \\
\sigma_{4} & :=\left(x_{1}, x_{2}, x_{3}\right) \rightarrow-\left(x_{2}, x_{1}, x_{3}\right) .
\end{aligned}
$$

Notice that $\sigma_{2}=\sigma_{3} \circ \sigma_{1} \circ \sigma_{3}$ and $\sigma_{4}=\sigma_{1} \circ \sigma_{3} \circ \sigma_{1}$. We call $G$ the group of symmetries of the Costa surface. In our case,

$$
G=\left\langle\sigma_{1}, \sigma_{3}\right\rangle .
$$

We remark that the Costa surface is invariant under a $180^{\circ}$-rotation around the $x_{3}$-axis. This rotation can be given by $\sigma_{1} \circ \sigma_{2}$. 


\section{Construction of the triply periodic Costa surfaces.}

We begin by considering the possibility of existence of triply periodic minimal surfaces in $\mathbb{R}^{3}$ fully generated by a fundamental piece $P$ which looks like a Costa surface with its planar end replaced by symmetry curves (see Figure 1(a)). We called $G$ the symmetry group of the Costa surface, which is isomorphic to the symmetry group of $P$.

The main goal of this paper is then to prove the following:

Theorem 4.1. There exists a one-parameter family of complete embedded triply periodic minimal surfaces in $\mathbb{R}^{3}$ such that, for any member of this family the following hold:

(a) The quotient by its translation group has genus 5 .

(b) The whole surface is generated by a fundamental piece $P$, which is a surface with border in $\mathbb{R}^{3}$. The border consists of four planar curves of vertical reflectional symmetry and two planar curves of horizontal reflectional symmetry. The fundamental piece has a symmetry group isomorphic to $G$, where $G$ is the symmetry group of the Costa surface.

(c) By successive reflections in the border of $P$ one obtains the triply periodic surface.

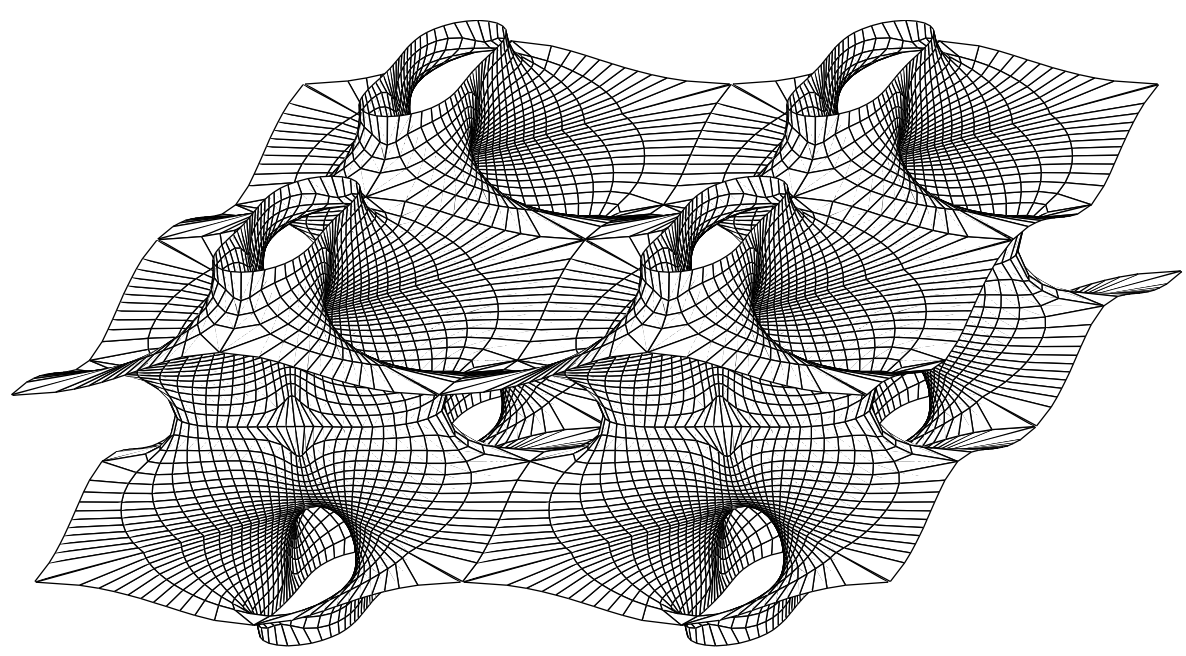

Figure 2. The triply periodic Costa surface for $\lambda=0.5$ (see below).

\section{The symmetries of the surface and the elliptic $Z$-function.}

From this point on we shall make use of heuristic arguments which will just help us in the formal demonstration of Theorem 4.1. Let us consider the surface represented in Figure 2. The quotient by its translation 
group generates a compact Riemann surface of genus 5 that we call $\bar{S}$ (see Figure 3(a)). Let $\rho$ be the quotient of $\bar{S}$ by its $180^{\circ}$-rotational symmetry around the $x_{3}$-axis. Then, the Euler-Poincaré characteristic of $\rho(\bar{S})$ is given by $\chi(\rho(\bar{S}))=\frac{\chi(\bar{S})}{2}+4=0$. Because of this, $\rho(\bar{S})$ is a torus that we call $T$. This torus must be rectangular because of the following argument. The horizontal reflectional symmetries of $\bar{S}$ are induced by $\rho$ in $T$. These turn out to be reflectional symmetries of $T$ as well, and there are two curves which remain invariant under any of these symmetries. Then, the fixed-point set has two components and this only happens for the rectangular torus.

The surface $\bar{S}$ has two other $180^{\circ}$-rotational symmetries, namely the ones around the $x_{1}$ - and $x_{2}$-axes. The torus $T$ has these two symmetries as well. Let $\widetilde{\rho}$ be the $180^{\circ}$-rotational symmetry around the $x_{1}$-axis. The quotient of $T$ by $\widetilde{\rho}$ is conformally $S^{2}$. After we fix an identification of $S^{2}$ with $\hat{\mathbb{C}}$, we finally obtain an elliptic function $Z: T \rightarrow S^{2}$. In the following we are going to use the same notation as in [1], pp. 170-171, to facilitate comparisons.

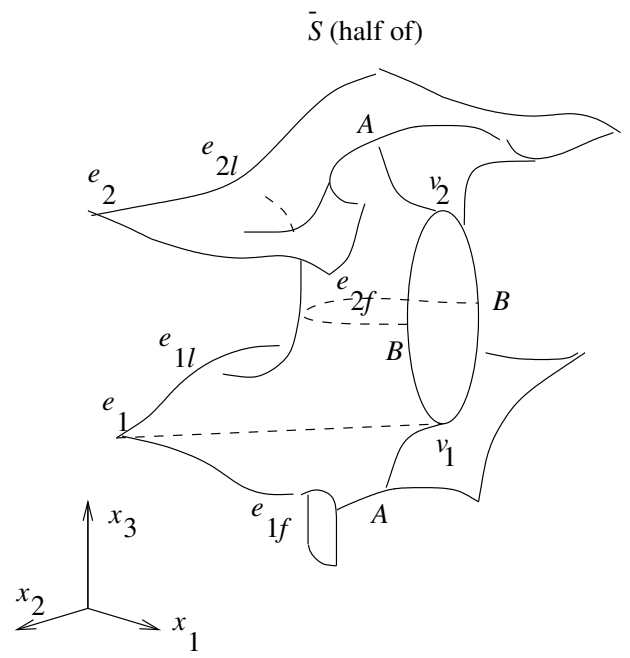

(a)

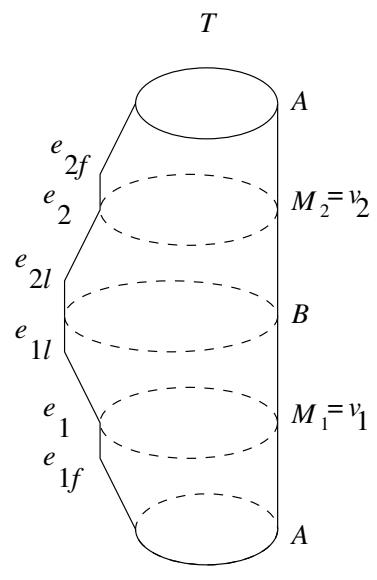

(b)

Figure 3. (a) Half of $\bar{S}$; (b) the torus $T=\rho(\bar{S})$.

Consider Figure 3(b) and the points of the torus $T$ represented there. These points correspond to special points of $\bar{S}$, represented on Figure 3(a) (they were given the same names). Let $Z: T \rightarrow S^{2}$ be the elliptic function with $Z(A)=\infty, Z\left(M_{1}\right)=Z\left(M_{2}\right)=-1$ and $Z(B)=0$ (this function is the same defined in [1], p. 171). The points $e_{1}$ and $e_{2}$ correspond to the ones in [1], p. 170, but in our case they will not lead to ends of the minimal surface.

On the surface $\bar{S}$, the unitary normal vector at $e_{1}, e_{2}, v_{1}, v_{2}$ is vertical. That is, $g+g^{-1}=\infty$. The other points at which $g+g^{-1}=\infty$ are labelled 
as $e_{1 l}, e_{1 f}, e_{2 l}, e_{2 f}$ ("l" and "f" mean "lateral" and "front"). In [1] , p. 170, one sees that the vertical points of the saddles, namely $v_{1}$ and $v_{2}$, do not coincide in general with the middle points $M_{1}$ and $M_{2}$ between $A$ and $B$, represented in Figure 4. But, in our case, the vertical reflectional symmetries of $\bar{S}$ imply $M_{2}=v_{2}$ and $M_{1}=v_{1}$.

Next we are going to summarize the important properties of the function $Z$ (see Figure 4).

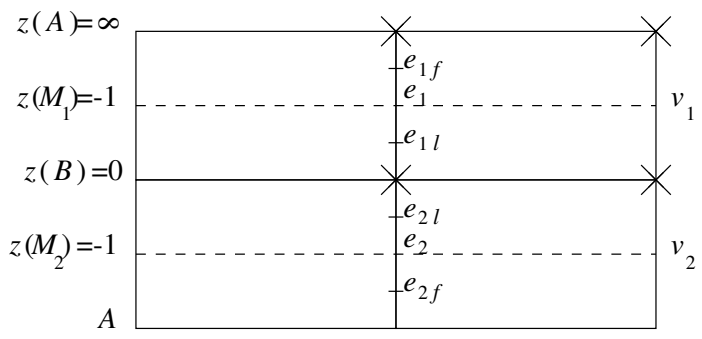

Figure 4. The torus $T$ with values of $Z$ at special points on it.

The function $Z$ is real on the bold lines (and nowhere else), and $|Z|=1$ on the dashed lines (and nowhere else). It has exactly four branch points, marked with $\times$ on Figure 4. Two of them are $A$ and $B$, where $Z$ assumes the values $\infty$ and 0 , respectively. At the centre $Z$ takes a value $\lambda \in(0,1)$ and at the other branch point it takes the value $\lambda^{-1}$. Let $x=Z\left(e_{1 l}\right)=Z\left(e_{2 l}\right)$, then $\lambda<x<1$. Now we write the most important values of $Z$ together:

$$
\begin{aligned}
Z\left(e_{1}\right) & =Z\left(e_{2}\right)=1, \\
Z\left(v_{1}\right) & =Z\left(v_{2}\right)=-1, \\
Z\left(e_{1 l}\right) & =Z\left(e_{2 l}\right)=x, \quad \text { and } \\
Z\left(e_{1 f}\right) & =Z\left(e_{2 f}\right)=x^{-1} .
\end{aligned}
$$

\section{The $z$-function on $\bar{S}$ and the Gauss map in terms of $z$.}

In this section we start by studying the necessary conditions for the existence of a minimal surface like in Figure 2. They will lead to an algebraic equation for the compact Riemann surface $\bar{S}$, together with Weierstrass data on it. From this point on, our problem will be concrete. We shall have to prove that the algebraic equation really corresponds to $\bar{S}$ in terms of its genus and symmetries. Afterwards, we shall have to prove that the Weierstrass data really lead to a minimal embedding of $\bar{S}$ in $\mathbb{R}^{3}$ with the desired properties: Symmetry curves, periodicity, etc.

Let us call $S$ the surface represented in Figure 2 and suppose that it is a minimal immersion of $\bar{S}$ in $\mathbb{R}^{3}$. In this case, we make use of the previous section and consider the functions $\rho: \bar{S} \rightarrow T$ and $Z: T \rightarrow \mathbb{C}$. Let us define 
$z:=Z \circ \rho$. Both functions $Z$ and $\rho$ have degree 2 , then $z$ is a function on $\bar{S}$ of degree 4 (see Figure 5(a)).

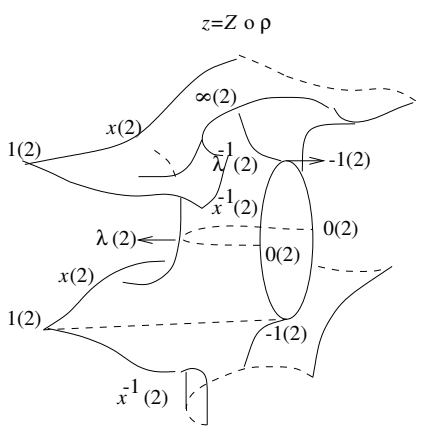

(a)

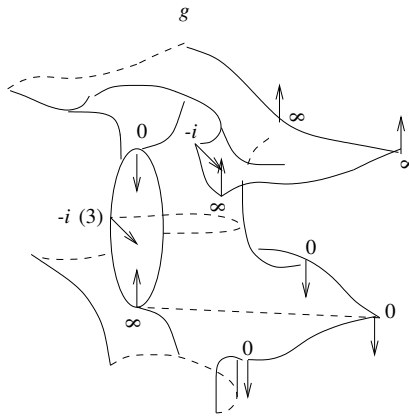

(b)

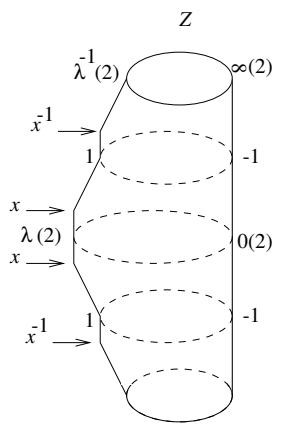

(c)

Figure 5. (a) Values of $z$ at special points; (b) the corresponding values of $g$ at these points; (c) the corresponding values of $Z$ on $T$.

We are supposing that $S$ is a minimal immersion of $\bar{S}$ in $\mathbb{R}^{3}$. In this case, the Gauss map on $S$ must lead to a meromorphic function $g$ on $\bar{S}$, as Figure 5(b) suggests. We are going to define multiplicity as the branch order plus one. Then, the expected correspondence between the values of $z$ and $g$ (including their multiplicities) is indicated in Figures 5(a) and 5(b). Notice that Figure 5 shows the particular case in which $g$ takes the values $\pm i$ and \pm 1 with multiplicity 3 at the points $z=0$. For this special case we expect the surface to have a four-fold symmetry saddle at these points.

In the general case, we need to introduce a new parameter. Consider $y \in(-1, \lambda)$ such that $z=y$ implies $g(z)= \pm i$ with multiplicity 1 . For $y=0$ the multiplicity is 3 (see Figure 5 ). The $180^{\circ}$-rotational symmetries correspond to the map $Z \rightarrow 1 / \bar{Z}$. Because of this, one has that $z=1 / y$ implies $g= \pm 1$. Based on what Figure 5 suggests and on this last remark we obtain the following relation between $g$ and $z$ :

$$
\left(g+\frac{1}{g}\right)^{2}=\frac{c z(z-y)^{2}\left(z-\lambda^{-1}\right)}{\left(z^{2}-1\right)(z-x)\left(z-x^{-1}\right)}
$$

where $c$ is a real positive constant. Notice that the functions $g$ and $z$ at both sides of (1) have the same poles and zeros, including their multiplicities, while $c, x, y$ and $\lambda$ are free parameters.

Now we have a concrete problem: We must show that (1) really represents the surface $\bar{S}$ in terms of its genus and symmetries. This will be true if the variables $c, x, y$ and $\lambda$ satisfy certain conditions which are going to be presented soon. During our demonstration of this fact we deduce that $c=4$. This is consistent with the expected position of the unitary normal vector at $z=\infty$ (see Figure 5(a)), which must correspond to $g= \pm 1$. 
Now we show that the function $g$ on $\bar{S}$, defined by (1) represents the Gauss map on the symmetry lines of the surface $S$. First of all, for the $180^{\circ}$-rotational symmetries of $S$, which correspond to $|z| \equiv 1$ on $\bar{S}$, we want $g^{2}$ to be pure imaginary. Define $X:=x+x^{-1}$. Then (1) leads to:

$$
g^{2}+g^{-2}=\frac{c(z-y)^{2}\left(1-\lambda^{-1} z^{-1}\right)-2\left(z-z^{-1}\right)\left(z-X+z^{-1}\right)}{\left(z-z^{-1}\right)\left(z-X+z^{-1}\right)} .
$$

On the right-hand side of (2) the denominator is pure imaginary. For $g^{2}+g^{-2}$ (and consequently $g^{2}$ ) to be pure imaginary as well we must have

$$
\operatorname{Im}\left\{c(z-y)^{2}\left(1-\lambda^{-1} z^{-1}\right)\right\}=\operatorname{Im}\left\{2\left(z-z^{-1}\right)\left(z-X+z^{-1}\right)\right\} .
$$

For $z=e^{i t}, t \in \mathbb{R},(3)$ leads to:

$$
c\left(2 \cos t-2 y-\lambda^{-1}+\lambda^{-1} y^{2}\right)=4(2 \cos t-X) .
$$

This is possible if, and only if $c=4$ and

$$
X=\frac{1+(2 \lambda-y) y}{\lambda}, \text { where } X:=x+x^{-1} .
$$

Recall that the variables $x, y$ and $\lambda$ must satisfy the following inequalities (see Figure 5(a)):

$$
-1<y<\lambda<x<1 \text { and } 0<\lambda .
$$

We can always choose $y$ and $\lambda$ with $-1<y<\lambda<1$ and $0<\lambda$ to get the value $x$ from (4). Nevertheless, the condition $x<1$ will not always be satisfied, unless $y>2 \lambda-1$ (we prove this assertion later). This will reduce the domain of $y$. In fact,

$$
y>2 \lambda-1 \text { implies } X>2 \text {, which implies } 0<x<1 \text {. }
$$

The remaining condition from (5), namely $\lambda<x$, is always valid for $0<x<1$ as a consequence of (4).

At this point we have shown that, under conditions (4) and $y>2 \lambda-1$, the phase of the function $g$ in (1) is constant and equals $\pm \pi / 4$ or $\pm 3 \pi / 4$ on the symmetry lines of the Riemann surface $\bar{S}$ which we want to be the straight lines on the minimal surface $S$. Now we are going to prove that the $180^{\circ}$-rotations around the straight lines lead to the expected change of the unitary normal vector. From Figures 5(a) and 5(b), these rotations suggest that, for example, $g=1$ is mapped to $g= \pm i$. Let us now verify this fact.

An important consequence of (4) is that it implies:

$$
4 z(z-y)^{2}\left(z-\lambda^{-1}\right)-4\left(z^{2}-1\right)(z-x)\left(z-x^{-1}\right)=4(1-y z)^{2}\left(1-\lambda^{-1} z\right) .
$$

With (2), (7) and the algebraic equality

$$
\left(g+g^{-1}\right)^{2}-4=\left(g-g^{-1}\right)^{2},
$$


one obtains:

$$
\left(g-\frac{1}{g}\right)^{2}=\frac{4(1-y z)^{2}\left(1-\lambda^{-1} z\right)}{\left(z^{2}-1\right)\left(z^{2}-X z+1\right)} .
$$

The $180^{\circ}$-rotational symmetries around the straight lines of the surface $S$ are represented by means of the map $z \rightarrow 1 / \bar{z}$ on $\bar{S}$ (notice that the points $|z|=1$ remain fixed). If we calculate $g(1 / \bar{z})$ from (8), we obtain:

$$
\left.\left(g-\frac{1}{g}\right)^{2}\right|_{1 / \bar{z}}=-\left.\left(\bar{g}+\frac{1}{\bar{g}}\right)^{2}\right|_{z}
$$

or equivalently, $g(1 / \bar{z})= \pm i \bar{g}(z)$. This means: The $180^{\circ}$-rotations of $S$ lead to the following maps for the function $g$ : either $g \rightarrow i \bar{g}$ or $g \rightarrow-i \bar{g}$. The fixed-point set of the former is given by $\left\{(z, g):|z|=1\right.$ and $\left.g=e^{i \frac{\pi}{4}}|g|\right\}$. For the latter, the fixed-point set is $\left\{(z, g):|z|=1\right.$ and $\left.g=e^{-i \frac{\pi}{4}}|g|\right\}$. Both maps correspond to an inversion of the surface orientation.

Now we prove that the compact Riemann surface (8) has genus 5 . From (1) and (8) we have that each value $z \in\left\{-1,1, x, x^{-1}, \lambda, \lambda^{-1}, 0, \infty\right\}$ represents 2 different branch points of order 1 (multiplicity 2 ) on the compact Riemann surface. The function $g$ is a four-sheet-covering and because of this, from the Riemann-Hurwitz formula the genus of $\bar{S}$ is:

$$
\frac{8 \cdot 2 \cdot(2-1)}{2}-4+1=5 .
$$

We still must show the following:

Proposition 6.1. Given $\lambda$ and $y$ in the interval $(-1,1)$ such that $y>2 \lambda-$ 1 , if $x$ and $c$ are determined by (4) then $x<1$.

Proof. This is a consequence of the equivalence of the following statements:

(a) $y>2 \lambda-1$;

(b) $(1-\lambda)^{2}>(\lambda-y)^{2}$;

(c) $2 \lambda<1+(2 \lambda-y) y$;

(d) $X>2$.

At this point we have proved that the correspondence between $z$ and $g$, given by (1) or (8), is consistent for the straight lines. Now we focus our attention on the remaining symmetry lines. Let us call $r^{2}$ the left-hand side of (1). Then, for every complex value $r$ and some branch of the square root one has:

$$
g^{-1}+g=r \text { implies } g=\frac{r \pm \sqrt{r^{2}-4}}{2} \text {. }
$$


Therefore, from (1) and based on Figure 5 we can briefly verify the values of $g$ on the planar symmetry curves as follows:

\begin{tabular}{|c|c|c|c|}
\hline 1 & $-1<z<0$ & $r \in i \mathbb{R}$ & $g \in i \mathbb{R}$ \\
\hline 2 & $-\infty<z<-1$ & $r \in(2, \infty)$ & $g \in \mathbb{R}$ \\
\hline 3 & $\lambda^{-1}<z<\infty$ & $r \in(0,2)$ & $|g|=1$ \\
\hline 4 & $x^{-1}<z<\lambda^{-1}$ & $r \in i \mathbb{R}$ & $g \in i \mathbb{R}$ \\
\hline 5 & $1<z<x^{-1}$ & $r \in \mathbb{R}$ & $g \in \mathbb{R}$ \\
\hline 6 & $x<z<1$ & $r \in i \mathbb{R}$ & $g \in i \mathbb{R}$ \\
\hline 7 & $\lambda<z<x$ & $r \in(2, \infty)$ & $g \in \mathbb{R}$ \\
\hline 8 & $0<z<\lambda$ & $r \in(0,2)$ & $|g|=1$ \\
\hline
\end{tabular}

We have just proved that the values of $g$ on all symmetry curves of the Riemann surface $\bar{S}$ are consistent with the expected unitary normal vector on the minimal surface $S$ in $\mathbb{R}^{3}$.

\section{The height differential $d h$ in terms of $z$.}

Now we need an expression for the differential form $d h$. The surface has no ends and because of this $d h$ is holomorphic. Its zeros are exactly the ones where $g=0$ or $g=\infty$ and all have multiplicity 1 (i.e., branch order 0 ). If we consider the differential form $d z$, then it would be sufficient to divide it by a function on the surface whose zeros were simple at $z \in\left\{0, \lambda, \lambda^{-1}\right\}$ and with a unique pole (of multiplicity 3 ) at $z=\infty$. This function will turn out to be the pull-back by $\rho$ of another function, that we call $V$, on the torus $T$.

Since $0, \lambda, \lambda^{-1}$ and $\infty$ are the only branch values of $Z$, all of them of order one, then the torus $T$ can be algebraically described by the equation $V^{2}=Z(Z-\lambda)\left(Z-\lambda^{-1}\right)$, and $V \circ \rho$ has exactly the zeros and poles on $\bar{S}$ with the desired multiplicities. We can define $v:=V \circ \rho$. This means that $v$ is a well-defined square root of the function $z\left(z-\lambda^{-1}\right)\left(1-\lambda^{-1} z\right)$ on $\bar{S}$. Another way to see this is to observe that (1) and (8) imply:

$$
z\left(z-\lambda^{-1}\right)\left(1-\lambda^{-1} z\right)=\frac{\left(z^{2}-1\right)^{2}\left(z^{2}-X z+1\right)^{2}}{16(z-y)^{2}(y z-1)^{2}}\left(g+\frac{1}{g}\right)^{2}\left(g-\frac{1}{g}\right)^{2} .
$$

Then, we can define

$$
\sqrt{z\left(z-\lambda^{-1}\right)\left(1-\lambda^{-1} z\right)}:=v=\frac{\left(z^{2}-1\right)\left(z^{2}-X z+1\right)}{4(z-y)(y z-1)}\left(g+\frac{1}{g}\right)\left(g-\frac{1}{g}\right) .
$$

Finally, we need to find a proportional constant to determine $d h$ by means of $\frac{d z}{v}$. On the straight lines of the surface, where $|z| \equiv 1$, the coordinate $x_{3}=\operatorname{Re} \int d h$ must be constant. Then $\operatorname{Re}\{d h\}$ is zero there. Because of this 
we choose the proportional constant to be 1 , namely

$$
d h=\frac{d z}{v}=\frac{d z}{\sqrt{z\left(z-\lambda^{-1}\right)\left(1-\lambda^{-1} z\right)}}=\frac{\lambda^{\frac{1}{2}} z^{-1} d z}{\sqrt{\lambda+\lambda^{-1}-z-z^{-1}}} .
$$

At this point we have reached concrete Weierstrass data $(g, d h)$ on $\bar{S}$, defined by (8) and (10), with $x, y$ and $\lambda$ satisfying (4) for $y$ in the interval $(2 \lambda-1, \lambda)$. Now our task will be the demonstration of the following: Let $S$ be the minimal immersion of $\bar{S}$ by these Weierstrass data. Then $S$ leads to the desired surface represented in Figure 2. In other words, we need to show that $S$ really has all the symmetry curves and lines of our initial assumptions and $S$ has no other periods except the ones indicated in Figure 2. This second task will be discussed in the next section. Now we analyse the symmetries of $S$.

From (9) and (10) we see that all $z$-curves listed in (9) are geodesics because $g(z)$ is contained either in a meridian or in the equator of $S^{2}$ and $d h(\dot{z})$ is contained in a meridian of $S^{2}$. Moreover, these geodesics are planar because $\frac{d g(\dot{z})}{g(z)} \cdot d h(\dot{z}) \in \mathbb{R}$. The expected straight lines of the surface, where $|z| \equiv 1$, come from (4) together with $y \in(2 \lambda-1, \lambda)$. We have already proved that (4) leads to $g /|g|=e^{ \pm i \pi / 4}$ on the straight lines. This means, $|z| \equiv 1$ implies $\frac{d g(\dot{z})}{g(z)} \cdot d h(\dot{z}) \in i \mathbb{R}$. Therefore, $S$ has all desired symmetries.

\section{Solution of the period problems.}

The quotient of the triply periodic minimal surface by its translation group leads to a compact surface $\bar{S}$. The left half of $\bar{S}$ is shown in Figure 6(a). The fundamental domain for the full symmetry group of the minimal surface is the shaded region represented on Figure 6(a).

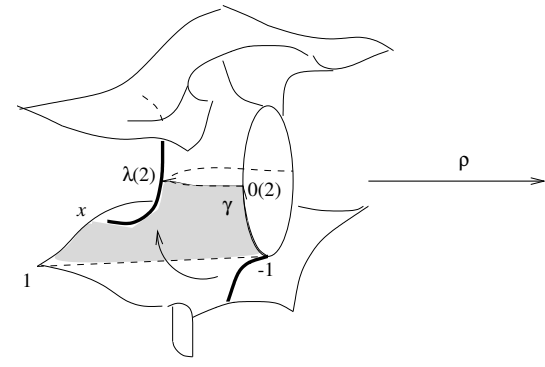

(a)

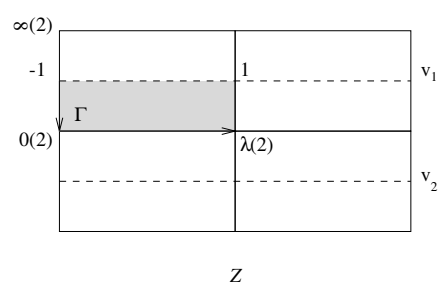

(b)

Figure 6. (a) The left half of $\bar{S}$; (b) its image under $\rho$.

We just need to analyse the period vector given by $\operatorname{Re} \oint\left(\phi_{1}, \phi_{2}, \phi_{3}\right)$ on the curves of the homology of $\bar{S}$. Let us consider the curves (1) to (8) from 
Table (9) as closed curves. The curve (1) crosses the planar geodesics (2) and (8), which are in orthogonal planes. Therefore, the period on (1) is zero. The curve (3) crosses the planar geodesics (2) and (4). Thus, the period on (3) is zero. The same conclusion is valid for (4), which crosses (3) and (5). The straight lines on $S$ bring (1), (3) and (4) respectively to (2), (8) and (7). Then, the periods on these three last curves is zero as well.

Nevertheless, (5) crosses orthogonally (4) and (6), which stay in planes parallel to $x_{1}=0$. Hence, (5) has a period exactly in the $x_{1}$-direction. Due to the straight lines on $S$, which interchange (5) and (6), the period on $(6)$ is exactly in the $x_{2}$-direction. Now consider Figure $3(\mathrm{~b})$ and the line segment which contains $A$ and $B$ represented there. Its inverse image by $\rho$ is a closed curve on $\bar{S}$ which crosses (3) and (8) orthogonally. Since (3) and (8) are parallel to $x_{3}=0$, the period on the curve will be exactly in the $x_{3}$-direction. A simple calculation can show us that the periods we are mentioning in this paragraph are not zero. But, if we show that they are the only periods of $S$, then the half-space theorem automatically guarantees that none of them is zero (see [5], p. 29).

Due to the straight lines on $S$, there remains just one curve of $\bar{S}$ on which we must analyse the period vector $\operatorname{Re} \oint\left(\phi_{1}, \phi_{2}, \phi_{3}\right)$. This curve we call $\gamma$ and it is represented on Figure 6(a). The curve $\gamma$ can be explicitly given by $z \circ \gamma(s)=s,-1<s<\lambda$. From Figure 6(a), we see that $\gamma$ crosses orthogonally the geodesics (1) and (7), which lie in planes parallel to $x_{2}=0$. Therefore, our task is reduced to the solution of the following equality:

$$
\operatorname{Re} \int_{\gamma} \phi_{2}=\operatorname{Re} \int_{\gamma} \frac{i}{2}\left(g+g^{-1}\right) d h=0 .
$$

To interpret (11) geometrically, consider the bold curves in Figure 6(a). They are supposed to belong to the same plane, and this condition is represented by (11). The integrand $\phi_{2}$ has two free parameters, namely $\lambda$ and $y$. If we fix the parameter $\lambda$, we can vary the $y$-parameter and try to make (11) valid. In other words, we then get the two bold curves in the same plane. Otherwise, they remain in distinct parallel planes.

Now observe Figure 6(b). We define $\Gamma:=\rho \circ \gamma$. Hence, $Z \circ \Gamma(s)=$ $z \circ \gamma(s)=s$. We need to calculate the integrand from (11) on $\gamma(s)$. To make this task easier, we split up both the curves $\gamma$ and $\Gamma$ into two pieces, one for $-1<s<0$ and the other for $0<s<\lambda$. The branches of the square root need to be chosen in accordance with Figures 6(a) and 6(b). Herewith we recall that the torus $T$ can be algebraically described by

$$
V^{2}=Z(Z-\lambda)\left(Z-\lambda^{-1}\right) .
$$

Let us define $\gamma_{1}(s)=\gamma(s), \Gamma_{1}(s)=\Gamma(s)$ for $-1<s<0$ and $\gamma_{2}(s)=$ $\gamma(s), \Gamma_{2}(s)=\Gamma(s)$ for $0<s<\lambda$. For the stretch $-1<s<0$ we take 
$s(t)=-t, 0<t<1$. Then, for $\gamma_{1}$ and $\Gamma_{1}$ we have:

$$
\begin{aligned}
\left.\left(\frac{1}{g}+g\right)^{2}\right|_{\gamma_{1}(s(t))} & =\frac{-4 t(t+y)^{2}\left(t+\lambda^{-1}\right)}{\left(1-t^{2}\right)(t+x)\left(t+x^{-1}\right)}<0,0<t<1 \\
V^{2}\left(\Gamma_{1}(s(t))\right) & =t^{2}\left(-\left(t+t^{-1}\right)-\left(\lambda+\lambda^{-1}\right)\right)<0 .
\end{aligned}
$$

We need to choose the square roots with signs compatible with the choice of the integration path represented on Figures 6(a) and 6(b). From now on consider positive the square root of positive reals. Now observe the following:

$$
-1=\frac{d}{d t}(Z(s(t)))=Z^{\prime}\left(\Gamma_{1}(t)\right) \cdot \Gamma_{1}^{\prime}(t) .
$$

This means that $Z^{\prime}\left(\Gamma_{1}(t)\right) \cdot \Gamma_{1}^{\prime}(t)$ is real. From Figure $6(\mathrm{~b})$ and Equations (13), our choice leads to $i \Gamma_{1}^{\prime}(t)>0$. Hence, one has $i Z^{\prime}\left(\Gamma_{1}(t)\right)>0$, namely, $Z^{\prime}(t)=-i \sqrt{t(t+\lambda)\left(t+\lambda^{-1}\right)}$.

Then $d h=\frac{-i d t}{Z^{\prime}(t)}>0,0<t<1$, which is in accordance with our choice indicated in Figures 6(a) and 6(b). Of course, this means that the $3^{\text {rd }}$ coordinate of our minimal surfaces $\left(x_{3}=\operatorname{Re} \int d h\right)$ is increasing along this path, which goes from $z=-1$ until $z=0$ (see Figure $6(\mathrm{a})$ ). But there are two different positions at which $z=0$ (at the back and at the front of the half piece). The one we want is at the front, as represented in Figure 6(a). There we see that on $\gamma_{1}$ the unitary normal vector on the surface leads to $-i g<0$. Then we choose our square root for $g^{-1}+g$ at (13) in such a way that $-i\left(g^{-1}+g\right) \circ \gamma_{1}>0$. Hence:

$$
\phi_{2}\left(\gamma_{1}(s(t))\right)=\frac{-(t+y) d t}{\sqrt{\left(1-t^{2}\right)(t+x)\left(t+x^{-1}\right)(t+\lambda)}} .
$$

For $\gamma_{2}$ and $\Gamma_{2}$ we take $s(t)=t, 0<t<\lambda$. Therefore:

$$
\left.\left(\frac{1}{g}+g\right)^{2}\right|_{\gamma_{2}(s(t))}=\frac{4 t(t-y)^{2}\left(\lambda^{-1}-t\right)}{\left(1-t^{2}\right)(x-t)\left(x^{-1}-t\right)}>0 .
$$

Based on (12) and Figure 6(b) we have that $\Gamma_{2}^{\prime}(t)>0$ and $\frac{d}{d t}(Z(t))=1$ imply $Z^{\prime}\left(\Gamma_{2}(t)\right)>0$. With an analogous argument as before we choose $g^{-1}+g<0$. Hence:

$$
\phi_{2}(\gamma(t))=\frac{(t-y) d t}{\sqrt{\left(1-t^{2}\right)(x-t)\left(x^{-1}-t\right)(\lambda-t)}} .
$$

At this point we are ready to write down (11) as an equality between two real integrals. But first we recall (see (4)):

$$
(-2 \lambda+y) y=1-\left(x+x^{-1}\right) \lambda \text { implies } y=\lambda-\sqrt{(x-\lambda)\left(x^{-1}-\lambda\right)} .
$$

We shall see that the period problem can be solved for every $\lambda$ in the interval $(0 ; 0.6+\varepsilon)$, where $\varepsilon>0$. Moreover, we shall see that the solution 
is unique for every fixed $\lambda$ in this interval. The theoretical value of $\varepsilon$ is not known yet, but numerical computations reach the approximate value of $\varepsilon=0.05$. Remember that $y \in(2 \lambda-1, \lambda)$ for $(5)$ to be valid. The following proposition is proved in [16], p. 3 :

Proposition 8.1. For a certain positive $\varepsilon<0.4$ one has that every $\lambda \in$ $(0 ; 0.6+\varepsilon)$ admits a unique $y_{\lambda} \in(2 \lambda-1, \lambda)$ such that, if $X_{\lambda}$ is the corresponding value of $x=x(\lambda, y)$ from $(4)$, then $\int_{0}^{1} \phi_{2}\left(\gamma_{1}(t)\right)+\int_{0}^{\lambda} \phi_{2}\left(\gamma_{2}(t)\right)=0$. Or equivalently, from (14) and (15):

$$
\int_{0}^{1} \frac{\left(t+y_{\lambda}\right) d t}{\sqrt{\left(1-t^{2}\right)\left(t^{2}+X_{\lambda} t+1\right)(t+\lambda)}}=\int_{0}^{\lambda} \frac{\left(t-y_{\lambda}\right) d t}{\sqrt{\left(1-t^{2}\right)\left(t^{2}-X_{\lambda} t+1\right)(\lambda-t)}} .
$$

In fact, it is possible to show that the period problem is unsolvable for $\lambda>0.8$, hence $\varepsilon<0.2$. Furthermore, one can prove the following: If $\varepsilon_{0}$ is the biggest value of $\varepsilon$, then the period problem is unsolvable for every $\lambda>0.6+\varepsilon_{0}$. In other words, the family of these triply periodic Costa surfaces is unique in the sense that there are no other subintervals of $(0,1)$, except $\left(0 ; 0.6+\varepsilon_{0}\right)$, in which one can find such surfaces. These results can be found in $[\mathbf{1 6}]$.

\section{Embeddedness of the triply periodic Costa surfaces.}

This section is strongly based on the ideas used in [9], pp. 60-62, where the author shows a demonstration for the embeddedness of the Costa surface in $\mathbb{R}^{3}$. We remark that now we have an explicit definition of $S$ (given by $g$ and $d h$ on $\bar{S}$ in (1)), and $S$ is represented in Figure 2 with no other periods but the ones suggested by the picture. In this section, we would like to verify if $S$ is embedded in $\mathbb{R}^{3}$. As we have already defined before, $\bar{S}$ is the quotient of $S$ by its translation group. Half of $\bar{S}$ is again reproduced in Figure 7(a). The shaded region indicated in this picture represents the fundamental domain of the surface, namely $\left(x_{1}, x_{2}, x_{3}\right)=\operatorname{Re} \int \phi_{1,2,3}:\{z \in \mathbb{C}:|z|<1$ and $\operatorname{Im}(z)>0\} \rightarrow \mathbb{R}^{3}$.

Now define $\mathcal{A}:=\{z \in \mathbb{C}:|z|<1$ and $\operatorname{Im}(z)>0\}$. By using the fact that $g$ is an open map of degree 4 , which corresponds to the unitary normal vector on the surface, it is then easy to verify the consistency of Figure 8. Since $g(\mathcal{A})$ is contained in a half-sphere, there is a direction in which the orthogonal projection of the fundamental domain is an immersion. In our case, we even have infinitely many such directions, but the most convenient is $\vec{x}_{2}$. Therefore, $\left(x_{1}, x_{3}\right): \mathcal{A} \rightarrow \mathbb{R}^{2}$ is an immersion. This is the first important argument for the demonstration of the embeddedness.

The orthogonal projection of $g(\mathcal{A})$ in the plane $x_{2}=0$ is represented on Figure 7(b). We want to prove that Figure 7(b) is consistent, namely, that the image curve of $z(t)=t, \lambda<t<x$, under the minimal immersion is well represented in Figure 7(b). Firstly, it has no intersections with the 


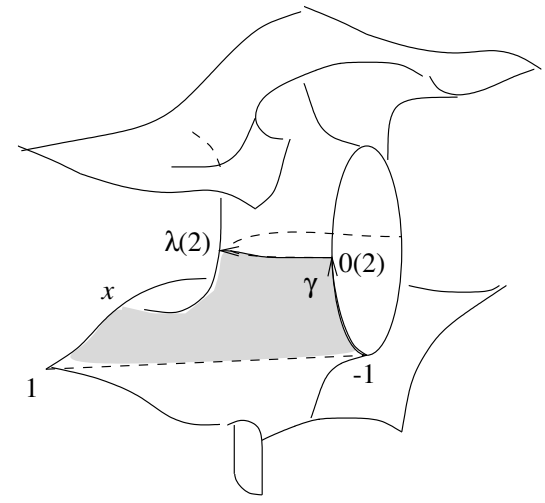

(a)

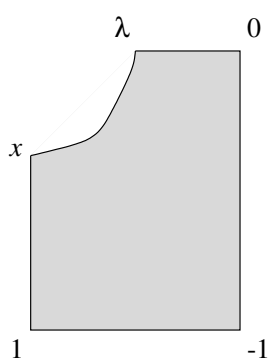

(b)

Figure 7. (a) The left half of $\bar{S}$; (b) the orthogonal projection of the fundamental domain on $x_{2}=0$.

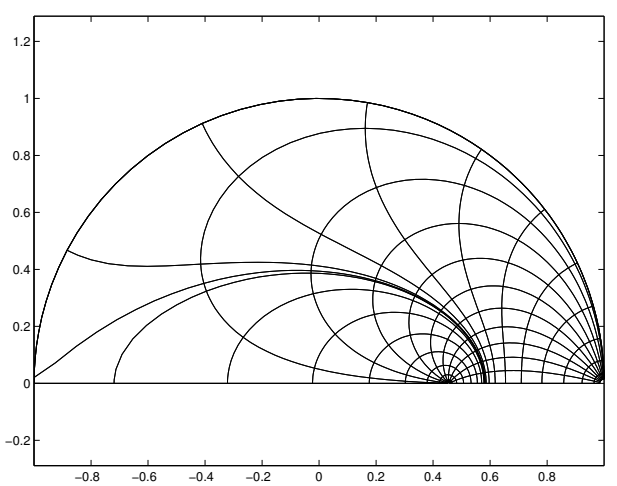

(a)

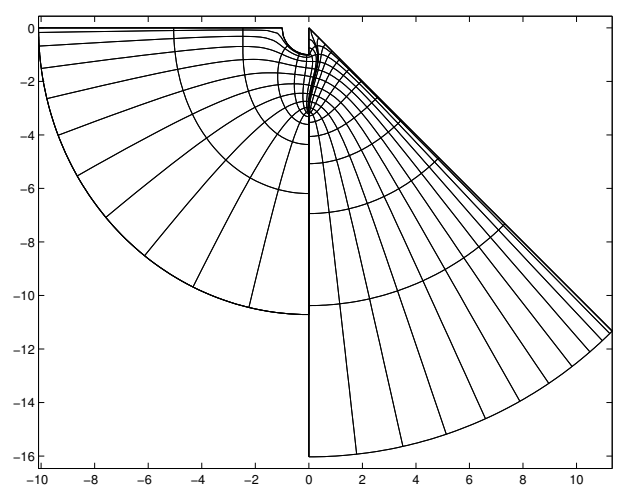

(b)

Figure 8. (a) The half-circle $\mathcal{A}$; (b) the corresponding image $g(\mathcal{A})$.

$x_{3}$-axis because $\left(x_{1}, x_{3}\right): \mathcal{A} \rightarrow \mathbb{R}^{2}$ is an immersion, hence open, and $\mathcal{A}$ is precompact. Secondly, the curve is convex because $g$ varies monotonely on it.

Simple calculations show that the other curves of the contour of $\left(x_{1}, x_{3}\right)(\mathcal{A})$ are monotone as well. Since $\left(x_{1}, x_{3}\right)$ is an immersion which fulfils the inside of this contour, and $\left(x_{1}, x_{3}\right)(\mathcal{A})$ is simply connected, it follows that $\left(x_{1}, x_{2}, x_{3}\right): \mathcal{A} \rightarrow \mathbb{R}^{3}$ is a graph. In particular, it is an embedding. One easily shows that the fundamental domain is inside a prism in $\mathbb{R}^{3}$. Together with a $180^{\circ}$-rotation around the straight line segment $\left(x_{1}, x_{3}\right)(\partial \mathcal{A} \backslash \mathbb{R})$, one gets an embedded piece of surface which is again inside a prism in $\mathbb{R}^{3}$ and whose border, now consisting only of reflectional symmetry curves, is contained in the border of the prism. By successive reflections in the border one 
obtains the whole triply periodic surface without self-intersections. Hence, it is an embedding because the immersion is proper.

Our next section will be devoted to the study of some limits of the triply periodic Costa surfaces.

\section{Limits of the triply periodic Costa surfaces family.}

In this section we are going to analyse what happens at the extremes of the triply periodic Costa surfaces family. In [16] we show the existence of a positive $\varepsilon_{0}<0.2$ such that the family exists only for $\lambda \in\left(0 ; 0.6+\varepsilon_{0}\right)$. Let us define $\lambda_{0}=0.6+\varepsilon_{0}$. We are going to show the following: If we fix the diameter of the closed curve (1) from Table (9), the convergence $\lambda \rightarrow \lambda_{0}$ implies that the triply periodic Costa surfaces converge to the $M_{3}$-CallahanHoffman-Meeks' surface in compact subsets of $\mathbb{R}^{3}$. If we fix the length of the straight line segments on $S$ which correspond to $|z| \equiv 1$, the convergence $\lambda \rightarrow 0$ implies that the triply periodic Costa surfaces converge to a pair of doubly periodic Scherk's surfaces in compact subsets of $\mathbb{R}^{3}$.

Let us first analyse the case $\lambda \rightarrow \lambda_{0}$. As demonstrated in [16], pp. 11-12, the functions

$$
\mathbb{I}_{1}(\lambda, y)=\int_{0}^{1} \frac{(t+y) d t}{\sqrt{\left(1-t^{2}\right)\left(t^{2}+X t+1\right)(t+\lambda)}}
$$

and

$$
\mathbb{I}_{2}(\lambda, y)=\int_{0}^{\lambda} \frac{(t-y) d t}{\sqrt{\left(1-t^{2}\right)\left(t^{2}-X t+1\right)(\lambda-t)}}
$$

are increasing and decreasing with $y$, respectively. As a matter of fact, it is a little bit more, namely: $\frac{\partial \mathbb{I}_{1}}{\partial y}>0$ and $\frac{\partial \mathbb{I}_{2}}{\partial y}<0$. Although we had restricted the $y$-domain to $(2 \lambda-1, \lambda)$, the functions $\mathbb{I}_{1}$ and $\mathbb{I}_{2}$ are defined for every $(\lambda, y) \in(0,1) \times(0,1)$. Of course, in this case we do not consider the variable $x$ with $x+x^{-1}=X$, but just $X=\frac{1+(2 \lambda-y) y}{\lambda}$, which can assume any positive value.

Now consider the function $\mathcal{F}:=\left(\mathbb{I}_{1}-\mathbb{I}_{2}\right):(0,1) \times(0,1) \rightarrow \mathbb{R}$. By Proposition 8.1 , for every $\lambda \in\left(0, \lambda_{0}\right)$ there exists a unique value $y_{\lambda}=y(\lambda)$ in the interval $(2 \lambda-1, \lambda)$ such that $\mathcal{F}(\lambda, y(\lambda))=0$. From the fact that $\frac{\partial \mathbb{I}_{1}}{\partial y}>0$ and $\frac{\partial \mathbb{I}_{2}}{\partial y}<0$, we have $\frac{\partial \mathcal{F}}{\partial y}>0$. Then, the implicit function theorem guarantees that the function $y_{\lambda}=y(\lambda)$ is differentiable. We can apply this theorem for $\mathcal{F}$ at $\left(\lambda_{0}, 2 \lambda_{0}-1\right)$. Let us define $I_{j}:=\mathbb{I}_{j}(\lambda, 2 \lambda-1), j=$ 1,2. From [16], pp. 15-16, we have that $I_{1}\left(\lambda_{0}\right)=I_{2}\left(\lambda_{0}\right)$. Therefore, $\mathcal{F}$ $\left(\lambda_{0}, 2 \lambda_{0}-1\right)=I_{1}\left(\lambda_{0}\right)-I_{2}\left(\lambda_{0}\right)=0$. By the implicit function theorem, there is a neighbourhood of $\lambda_{0}$ in which one can define $y=y(\lambda)$, such that $\mathcal{F}$ $(\lambda, y(\lambda))=0$ for every $\lambda$ in this neighbourhood. Due to the uniqueness of 
$y_{\lambda}$, we conclude that:

$$
\lim _{\lambda \rightarrow \lambda_{0}} y(\lambda)=2 \lambda_{0}-1
$$

Because of (4) and (16), we conclude that $\lambda \rightarrow \lambda_{0}$ implies $x \rightarrow 1$. Now consider the algebraic Equation (1) of the compact Riemann surface $\bar{S}$, which associates the Gauss map $g$ on $S$ and the meromorphic function $z$ :

$$
\left(g+\frac{1}{g}\right)^{2}=\frac{4 z(z-y)^{2}\left(z-\lambda^{-1}\right)}{\left(z^{2}-1\right)(z-x)\left(z-x^{-1}\right)} .
$$

Let us take a compact set $\mathcal{K} \subset \bar{A}=\{z \in \mathbb{C}:|z| \leq 1$ and $\operatorname{Im}(z) \geq 0\}$. This set is contained in the upper half of the unitary disk in $\mathbb{C}$, where we can consider $z$ as a variable on it, $g$ and $d h / d z$ as functions of $z$. We are going to fix the diameter of the closed curve (1) from Table (9), whose length is given by

$$
d(\lambda)=2 \int_{z(t)} d h(\dot{z}(t)), z(t)=t,-1<t<0 .
$$

Therefore, we are going to work with the Weierstrass data $\left(g, d^{-1} d h\right)$ on the compact set $\mathcal{K} \subset \mathbb{C}$. Choose any point $p_{0} \in \mathcal{K} \backslash\{1\}$ and for every $p \in \mathcal{K} \backslash\{1\}$ an integrable curve in $\mathcal{K} \backslash\{1\}$ connecting $p_{0}$ and $p$. Of course, the integrands $\phi_{j}, j \in\{1,2,3\}$ have singularities at $z \in\{-1,0, \lambda\}$, but the changes of variable $z \rightarrow 1+z^{2}, z \rightarrow z^{2}$ and $z \rightarrow z^{2}-\lambda$ can be used to make them bounded in $\mathcal{K}$. Hence, the coordinate functions $\left(x_{1}, x_{2}, x_{3}\right)_{\lambda}(p)=\operatorname{Re} \int_{p_{0}}^{p}\left(\phi_{1}, \phi_{2}, \phi_{3}\right)$ are uniformly bounded in $\mathbb{R}^{3}$, for every $\lambda$ in a neighbourhood of $\lambda_{0}$. We can fix this neighbourhood to be $\left(\frac{1}{2}, \lambda_{0}\right)$. Then, the coordinates are inside a compact subset of $\mathbb{R}^{3}$, for every $\lambda \in\left(\frac{1}{2}, \lambda_{0}\right)$.

Moreover, a simple calculation shows that the convergence of the coordinate functions $\left(x_{1}, x_{2}, x_{3}\right)_{\lambda} \rightarrow\left(x_{1}, x_{2}, x_{3}\right)_{\lambda_{0}}$ is uniform on $\mathcal{K}$. Let us then analyse the special case $\lambda=\lambda_{0}, y=2 \lambda_{0}-1$ and $x=1$ for (1). In this case we have:

$$
\left(g+\frac{1}{g}\right)^{2}=\frac{4 z\left(z-2 \lambda_{0}+1\right)^{2}\left(z-\lambda_{0}^{-1}\right)}{(z+1)(z-1)^{3}} .
$$

By the Riemann-Hurwitz formula, Equation (18) represents a compact Riemann surface of genus 3 , which we call $\bar{S}_{\lambda_{0}}$. Table (9) remains valid for $\bar{S}_{\lambda_{0}}$ (of course, the stretches $x<z<1$ and $1<z<x^{-1}$ must not be considered). This means, except for these stretches, all the other symmetries of the triply periodic Costa surfaces are symmetries of $\bar{S}_{\lambda_{0}}$ as well. In particular, $\bar{S}_{\lambda_{0}}$ has a $180^{\circ}$-rotational symmetry around the $x_{3}$-axis. The left half of $\bar{S}_{\lambda_{0}}$ is represented in Figure 9(a) (compare Figure 9 with Figure 5). 


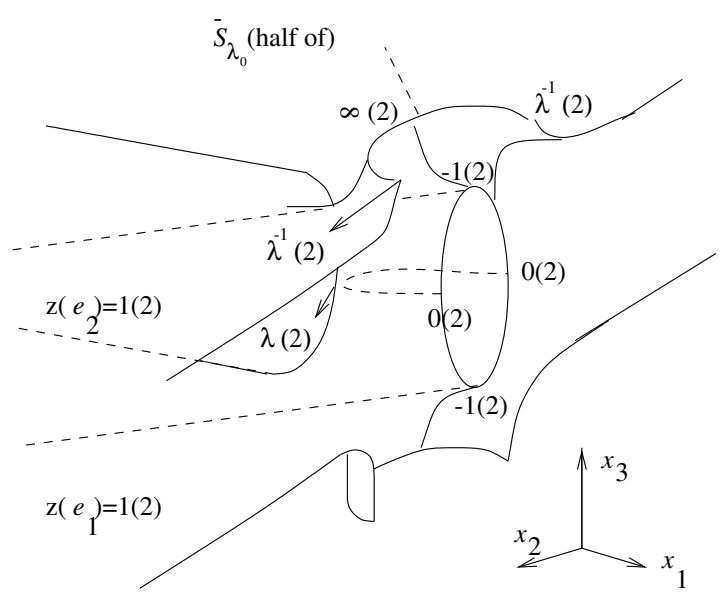

(a)

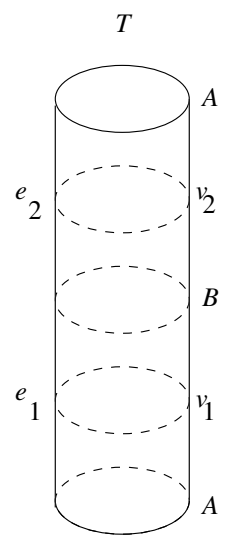

(b)

Figure 9. (a) The left half of $\bar{S}_{\lambda_{0}}$ with $z=x=1$ at infinity; (b) the torus $T=\rho\left(\bar{S}_{\lambda_{0}}\right)$.

If $\rho$ is the projection of $\bar{S}_{\lambda_{0}}$ by the $180^{\circ}$-rotational symmetry around the $x_{3}$-axis, then the Euler-Poincaré characteristic of $\rho\left(\bar{S}_{\lambda_{0}}\right)$ is given by

$$
\chi\left(\rho\left(\bar{S}_{\lambda_{0}}\right)\right)=\frac{\chi\left(\bar{S}_{\lambda_{0}}\right)}{2}+2=0 .
$$

Then $\rho\left(\bar{S}_{\lambda_{0}}\right)$ is a torus that we call $T$ as well. The surface $\bar{S}_{\lambda_{0}}$ has reflectional symmetries whose fixed point set consists of two components. After the projection $\rho$, the torus $T$ will also have these symmetries, with the fixed point set consisting of two components. Therefore, $T$ must be a rectangular torus.

Some special values of $z=Z \circ \rho$ are represented on Figure 9(a), together with their multiplicity. Now, the Gauss map $g$ and the differential holomorphic form:

$$
d h=\frac{d z}{v}=\frac{d z}{\sqrt{z\left(z-\lambda_{0}^{-1}\right)\left(1-\lambda_{0}^{-1} z\right)}},
$$

constitute a Weierstrass pair $(g, d h)$ on $\bar{S}_{\lambda_{0}}$. At the point $z=1$, on the one hand we have $g=0$ or $g=\infty$ (both of multiplicity 3). On the other hand, $d h=0$ at this point. This characterizes a planar end. In Section 9 we saw that every member of the triply periodic Costa surfaces family is embedded in $\mathbb{R}^{3}$. In the same way as in Section 9 , one can prove that the minimal immersion of $\bar{S}_{\lambda_{0}} \backslash z^{-1}(\{1\})$ in $\mathbb{R}^{3}$, defined by $(g, d h)$, is embedded as well. The fact that this embeddedness leads to the $M_{3}$-Callahan-Hoffman-Meeks' 
surface comes from the following proposition, which is due to Francisco Martín and Domingo Rodríges (see [11]):

Proposition 10.1. Let $M$ be a properly embedded minimal surface in $\mathbb{R}^{3}$ satisfying the following:

1. $M$ has an infinite number of annular ends;

2. $M$ is invariant under a cyclic group of translations $\tau$;

3. $M / \tau$ has genus $k \geq 2$ and 2 ends;

4. $|\operatorname{Iso}(M / T)| \geq 4(k+1)$.

Then, $k$ is odd and $M$ is the $M_{k}$-Callahan-Hoffman-Meeks' surface.

Now we are going analyse the consistence of our results with [2]. The numeric value of $\lambda_{0}$ for our surface $S_{\lambda_{0}}$ is approximately 0.65 (see Figure 12). In [2], the period problem is solved with means of another parameter whose value is approximately 0.4 . We want to relate these two parameters and verify if their relation is consistent with these values.

Consider Figure 10(a) which represents the left half of $M_{3}$ divided by its vertical translation $\tau$. The surface $M_{3}$ is invariant under the $180^{\circ}$-rotational symmetry around the $x_{3}$-axis, and because of the same arguments used for $\rho\left(\bar{S}_{\lambda_{0}}\right)$, this torus is rectangular. We are going to represent this torus by $\mathcal{T}$. Since $M_{3}$ has two other $180^{\circ}$-rotational symmetries, namely the ones around the $x_{1}$ - and $x_{2}$-axes, the torus $\mathcal{T}$ has these two symmetries as well. Let $\widetilde{\rho}$ be its $180^{\circ}$-rotational symmetry around the $x_{1}$-axis. The quotient of $\mathcal{T}$ by $\widetilde{\rho}$ is conformally $S^{2}$. After we fix an identification of $S^{2}$ with $\hat{\mathbb{C}}$, this will define an elliptic function $U: \mathcal{T} \rightarrow S^{2}$.

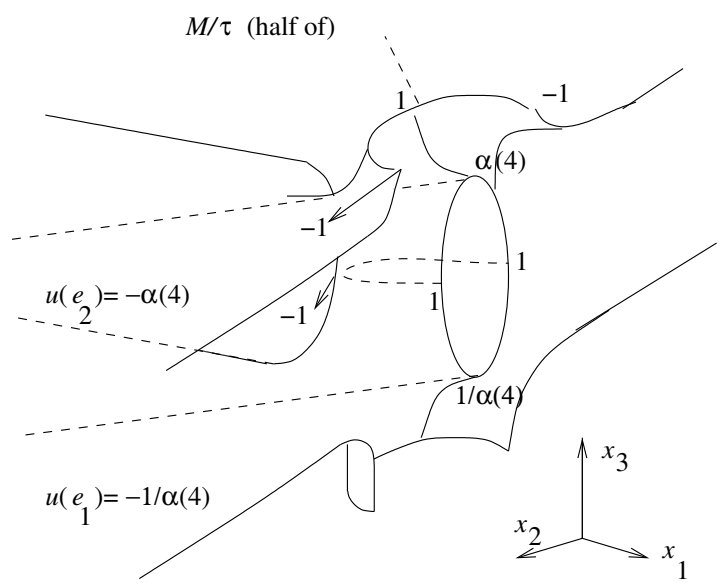

(a)

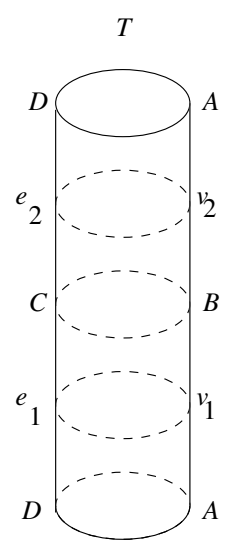

(b)

Figure 10. (a) The left half of $M_{3} / \tau$; (b) the torus $\mathcal{T}=\rho\left(\bar{S}_{\lambda_{0}}\right)$. 
Consider Figure 10(b) and the points of the torus $\mathcal{T}$ represented there. These points correspond to special points of $M_{3} / \tau$, represented on Figure 10(a) (they were given the same names). Let $U: \mathcal{T} \rightarrow S^{2}$ be the elliptic function with $U(A)=U(B)=1$ and $U\left(v_{1}\right)=1 / U\left(v_{2}\right)=\alpha$, where $\alpha$ is a real value in $(0,1)$ (these functions are the same functions $z$ defined in [2], p. 501).

Next we are going to summarize some important properties of the function $U$ (see Figure 11).

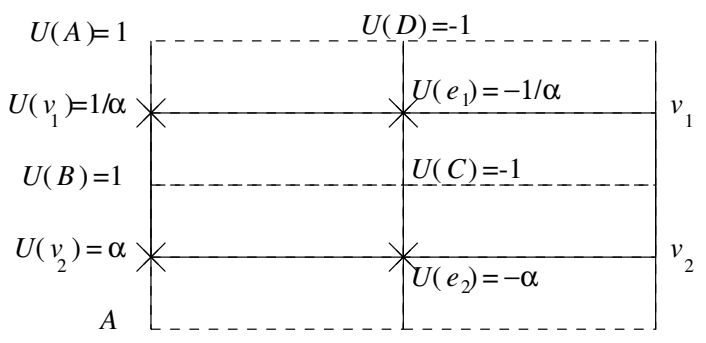

Figure 11. The torus $\mathcal{T}$ with values of $U$ at special points on it.

The function $U$ is real on the bold lines (and nowhere else), and $|U|=1$ on the dashed lines (and nowhere else). It has exactly four branch points, marked with $\times$ on Figure 11. At the points $C$ (centre) and $D, U$ takes the value -1 . At the other branch points $e_{1}$ and $e_{2}$, it takes the values $-\alpha^{-1}$ and $-\alpha$, respectively. Now we write the most important values of $U$ together:

$$
\begin{aligned}
U(A) & =U(B)=1, \\
U(C) & =U(D)=-1, \\
U\left(v_{2}\right) & =1 / U\left(v_{1}\right)=\alpha, \quad \text { and } \\
U\left(e_{2}\right) & =1 / U\left(e_{1}\right)=-\alpha .
\end{aligned}
$$

On the surface $M_{3} / \tau$, the unitary normal vector at $e_{1}, e_{2}, v_{1}, v_{2}$ is vertical, that is, $g\left(v_{2}\right)=g\left(e_{2}\right)=0$ and $g\left(v_{1}\right)=g\left(e_{1}\right)=\infty$. From Theorem 2.5 we have

$$
\operatorname{deg}(g)=3+2-1=4 .
$$

Let us define the function $u:=U \circ \rho$ on $M_{3} / \tau$. The most important values of $u$ on $M_{3} / \tau$ are indicated on Figure 10(a). Based on Figure 10(a), the following is the relation which must hold between the Gauss map and $u$ :

$$
g^{4} \sim \frac{(u-\alpha)(u+1 / \alpha)^{3}}{(u-1 / \alpha)(u+\alpha)^{3}} .
$$

This algebraic equation is the same as in [2], p. 502 (our parameter $\alpha$ and our function $u$ are represented there by " $\lambda$ " and " $z$ ", respectively). Since 
inversion through $|u|=1$ is an isometry for $M_{3}$, the proportional constant at (20) must be $\alpha^{2}$ (this is the same argument from [2], p. 503).

By the same reasons explained for $T$, the torus $\mathcal{T}$ can be algebraically described by

$$
U^{\prime 2}=\left(U^{2}-\alpha^{2}\right)\left(U^{2}-1 / \alpha^{2}\right) .
$$

Since $M_{3} / \tau$ has just two ends, both planar, the height-differential $d h$ for the Weierstrass pair $(g, d h)$ on $M_{3} / \tau$ must be:

$$
d h \sim \frac{d u}{U^{\prime} \circ \rho} .
$$

On the straight lines of the surface, $\operatorname{Re} \int d h$ must be constant. On these lines, $U^{\prime}$ is imaginary and $u$ is real. Because of this, we choose the proportional constant in (21) to be 1.

At this point, we would like to comment that there is a unique $\alpha \in(0,1)$ which solves the period problem for $M_{3}$. This fact is not proved in [2], but in $[\mathbf{1 1}]$.

Remark. The tori $T$ and $\mathcal{T}$ are biholomorphic if their cross ratios are equal. In other words, if there is a Möbius transformation $w: S^{2} \rightarrow S^{2}$ such that $Z=w \circ U$. On the one hand, an algebraic equation for $T$ is

$$
V^{2}=Z\left(Z-\lambda_{0}\right)\left(Z-\lambda_{0}^{-1}\right)
$$

Therefore, the cross ratio of $T$ is

$$
\frac{\lambda_{0}^{-1}-\infty}{\lambda_{0}^{-1}-0} \cdot \frac{\lambda_{0}-0}{\lambda_{0}-\infty}=\lambda_{0}^{2} .
$$

On the other hand, the cross ratio of $\mathcal{T}$ is

$$
\frac{1 / \alpha+1 / \alpha}{1 / \alpha+\alpha} \cdot \frac{\alpha+\alpha}{\alpha+1 / \alpha}=\frac{4 \alpha^{2}}{\left(1+\alpha^{2}\right)}
$$

Because of this, the variables $\lambda_{0}$ and $\alpha$ must fulfil the following condition:

$$
\lambda_{0}=\frac{2 \alpha}{1+\alpha^{2}} \text {. }
$$

From Proposition 10.1, Equations (18) and (19) lead to the $M_{3}$-CallahanHoffman-Meeks' surface. Therefore, (22) holds. In [2], p. 503, there is a graph which indicates that $\alpha \cong 0.4$. This implies that $\lambda_{0} \cong 0.65$ and vice versa, which is consistent with Figure 12.

Now we are going to analyse the case $\lambda \rightarrow 0$. This is the last task of this section. From $[\mathbf{1 6}]$, p. 3, we have

$$
\lim _{\lambda \rightarrow 0} \mathbb{I}_{1}(\lambda, \lambda)=\lim _{\lambda \rightarrow 0} \mathbb{I}_{2}(\lambda, \lambda)=0 .
$$




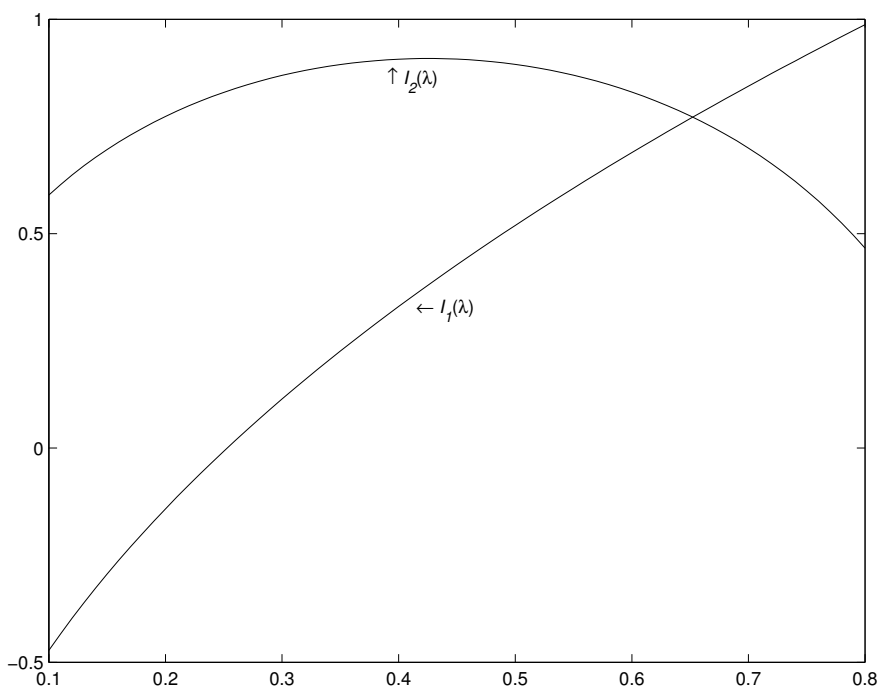

Figure 12. Numeric values for $I_{1}$ and $I_{2}$.

From [16], p. 13, one concludes that

$$
\lim _{\lambda \rightarrow 0} \mathbb{I}_{1}(\lambda, 2 \lambda-1)=-\infty \text { and } \lim _{\lambda \rightarrow 0} \mathbb{I}_{2}(\lambda, 2 \lambda-1)=0 .
$$

We have proved in $[\mathbf{1 6}]$, pp. 11-12, that $\mathbb{I}_{1}(\lambda, y)$ is increasing while $\mathbb{I}_{2}(\lambda, y)$ is decreasing with $y$. From (23) and (24) we conclude that

$$
\lim _{\lambda \rightarrow 0} y(\lambda)=0 .
$$

From (4) and (25) it follows that $\lambda \rightarrow 0$ implies $X \rightarrow \infty$ and consequently $x \rightarrow 0$. We want to write down (1) for the special case $\lambda=x=y=0$. From (4) and (25) one easily sees that $\lambda \rightarrow 0$ implies $x^{-1} \lambda \rightarrow 0$. Because of this, the case $\lambda \rightarrow 0$ for (1) leads to

$$
\left(g+\frac{1}{g}\right)^{2}=\frac{4 z^{2}}{z^{2}-1},
$$

which we rewrite, for instance, as

$$
g^{2}=\frac{z+1}{z-1} .
$$

Of course, we are again considering $z$ as a variable on the compact set $\mathcal{K}$ with $g$ and $d h / d z$ as functions of $z$. We are going to fix the length of the straight line segment on $S$ which corresponds to the image in $\mathbb{R}^{3}$ of the curve $z(t)=e^{i t}, t \in[0, \pi]$. This length is given by

$$
l(\lambda)=\sqrt{2} \cdot\left|\int_{0}^{1} \phi_{1}(z(t))\right|,
$$


and it is easy to see that $L_{0}:=\lim _{\lambda \rightarrow 0} \frac{l(\lambda)}{\lambda}$ is positive and finite. Recalling (1) and $(10)$, we are going to work with the Weierstrass data $\left(g, \frac{L_{0}}{l(\lambda)} d h\right)$ on the compact set $\mathcal{K} \subset \mathbb{C}$. From (10) we have that the differential $\lambda^{-1} d h$ for $\lambda \rightarrow 0$ is:

$$
d h=\frac{d z}{z} .
$$

Choose any point $p_{0} \in \mathcal{K} \backslash \mathbb{R}_{+}$and for every $p \in \mathcal{K} \backslash \mathbb{R}_{+}$an integrable curve in $\mathcal{K} \backslash \mathbb{R}_{+}$connecting $p_{0}$ and $p$. Then, the coordinate functions $\left(x_{1}, x_{2}, x_{3}\right)_{\lambda}(p)=\operatorname{Re} \int_{p_{0}}^{p}\left(\phi_{1}, \phi_{2}, \phi_{3}\right)$ are uniformly bounded in $\mathbb{R}^{3}$, for every $\lambda$ in a neighbourhood of 0 . We can fix this neighbourhood to be $\left(0, \frac{1}{2}\right)$. Then, the coordinates are inside a compact subset of $\mathbb{R}^{3}$, for every $\lambda \in\left(0, \frac{1}{2}\right)$. A simple calculation shows that the convergence $\left(x_{1}, x_{2}, x_{3}\right)_{\lambda} \rightarrow\left(x_{1}, x_{2}, x_{3}\right)_{0}$ is uniform on $\mathcal{K}$.

Applying the Möbius transformation $z=\frac{w+1}{w-1}$ we get

$$
g^{2}=w \text { and } d h=\frac{-2 d w}{w^{2}-1}=\frac{-4 d g / g}{g^{2}-g^{-2}},
$$

which are the the Weierstrass data on the sphere $g^{2}=w$ for the doubly periodic Scherk's surface. This concludes our last section.

Acknowledgements. This work refers to part of my doctoral thesis [15], which was supported by DAAD - Deutscher Akademischer Austausch Dienst, and CAPES - Coordenação de Aperfeiçoamento de Pessoal de Nível Superior. My adviser was Prof. Hermann Karcher, from the University of Bonn (Germany). I thank him for his dedication, which much helped in the realisation of my work.

\section{References}

[1] M. Callahan, D. Hoffman and H. Karcher, A family of singly periodic minimal surfaces invariant under a screw motion, Experimental Math., 2(3) (1993), 157-182, MR 95h:53007, Zbl 0807.53004.

[2] M. Callahan, D. Hoffman and W. Meeks, Embedded minimal surfaces with an infinite number of ends, Invent. Math., 96 (1989), 459-505, MR 90b:53005, Zbl 0676.53004.

[3] C. Costa, Example of a complete minimal immersion in $\mathbb{R}^{3}$ of genus one and three embedded ends, Bol. Soc. Bras. Mat., 15 (1984), 41-54, MR 87c:53111, Zbl 0613.53002.

[4] K. Große-Brauckmann and M. Wohlgemuth, The gyroid is embedded and has constant mean curvature companions, Calc. Var. Partial Differential Equations, 6(4) (1996), 499-523, MR 97k:53011, Zbl 0930.53009.

[5] D. Hoffman and H. Karcher, Complete embedded minimal surfaces of finite total curvature, Encyclopaedia of Math. Sci., 90, Springer Verlag, Berlin, 1997, 5-93, MR 98m:53012, Zbl 0890.53001. 
[6] D. Hoffman and W. Meeks, A complete embedded minimal surface in $\mathbb{R}^{3}$ with genus one and three ends, J. Differential Geom., 21 (1985), 109-127, MR 87d:53008, Zbl 0604.53002.

[7] _ Embedded minimal surfaces of finite topology, Ann. of Math., 131 (1990), 1-34, MR 91i:53010, Zbl 0695.53004.

[8] A. Huber, On subharmonic functions and differential geometry in the large, Comment. Math. Helv., 32 (1957), 13-72, MR 20 \#970, Zbl 0080.15001.

[9] H. Karcher, Construction of minimal surfaces, Surveys in Geometry, University of Tokyo, 1989, 1-96, and Lecture Notes, SFB256 Bonn, 12, 1989.

[10] - The triply periodic minimal surfaces of Alan Schoen and their constant mean curvature companions, Manuscripta Math., 64 (1989), 291-357, MR 90g:53010, Zbl 0687.53010.

[11] F. Martín and D. Rodrígues, A characterization of the periodic Callahan-HoffmanMeeks surfaces in terms of their symmetries, Duke Math. J., 89(3) (1997), 445-463, MR 98h:53016, Zbl 0901.53006.

[12] J.C.C. Nitsche, Lectures on Minimal Surfaces, Second edition, Dover, New York, 1986, MR 90m:49031, Zbl 0688.53001.

[13] R. Osserman, Global properties of minimal surfaces in $E^{3}$ and $E^{n}$, Ann. of Math. (2), 80 (1964), 340-364, MR 31 \#3946, Zbl 0134.38502.

[14] _ A Survey of Minimal Surfaces, Second edition, Dover, New York, 1986, MR 87j:53012, Zbl 0209.52901.

[15] Construction of New Complete Minimal Surfaces in $\mathbb{R}^{3}$ Based on the Costa Surface, Doctoral Thesis, University of Bonn, 2000.

[16] _ Theoretical evaluation of elliptic integrals based on computer graphics, UNICAMP Technical Report 71/02, Campinas, SP 2002; home page http://www.ime.unicamp.br/rel'pesq/2002/rp71-02.html.

[17] A.H. Schoen, Infinite periodic minimal surfaces without selfintersections, NASA Technical Note D-5541, Cambridge, MA, 1970.

[18] H.A. Schwarz, Gesammelte Mathematische Abhandlungen, Springer Verlag, Berlin, 1890, MR 52 \#13287, Zbl 22.0031.04.

Received April 26, 2002 and revised December 9, 2002.

Instituto de Mathemática, Estatística e ComputaÇão Científica

CP 6065

13089-859 CAMPINAS SP

BRAZIL

E-mail address: valerio@ime.unicamp.br 